\title{
Numerical Investigation of Local Heat-Release Rates and Thermo-Chemical States in Side-Wall Quenching of Laminar Methane and Dimethyl Ether Flames
}

\author{
M. Steinhausen ${ }^{1}$ (D) Y. Luo ${ }^{1}$. S. Popp ${ }^{1} \cdot$ C. Strassacker ${ }^{2} \cdot$ T. Zirwes ${ }^{3,4}$ (D) H. Kosaka ${ }^{5}$.

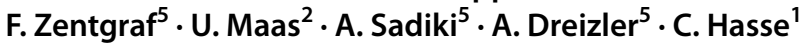

Received: 9 December 2019 / Accepted: 14 April 2020 / Published online: 13 May 2020

(C) The Author(s) 2020

\begin{abstract}
The local heat-release rate and the thermo-chemical state of laminar methane and dimethyl ether flames in a side-wall quenching configuration are analyzed. Both, detailed chemistry simulations and reduced chemistry manifolds, namely Flamelet-Generated Manifolds (FGM), Quenching Flamelet-generated Manifolds (QFM) and Reaction-Diffusion Manifolds (REDIM), are compared to experimental data of local heat-release rate imaging of the lab-scale side-wall quenching burner at Technical University of Darmstadt. To enable a direct comparison between the measurements and the numerical simulations, the measurement signals are computed in all numerical approaches. Considering experimental uncertainties, the detailed chemistry simulations show a reasonable agreement with the experimental heat-release rate. The comparison of the FGM, QFM and REDIM with the detailed simulations shows the high prediction quality of the chemistry manifolds. For the first time, the thermo-chemical state during quenching of a dimethyl ether-air flame is examined numerically. Therefore, the carbon monoxide and temperature predictions are analyzed in the vicinity of the wall. The obtained results are consistent with previous studies for methane-air flames and extend these findings to more complex oxygenated fuels. Furthermore, this work presents the first comparison of the QFM and the REDIM in a side-wall quenching burner.
\end{abstract}

Keywords Flame-wall interaction · Side-wall quenching $\cdot$ REDIM $\cdot$ FGM $\cdot$ QFM $\cdot$ DME

M. Steinhausen

steinhausen@stfs.tu-darmstadt.de

1 Simulation of reactive Thermo-Fluid Systems, Technical University of Darmstadt, Otto-Berndt-Str. 2, 64287 Darmstadt, Germany

2 Institute of Technical Thermodynamics, KIT, Engelbert-Arnold-Str. 4, 76131 Karlsruhe, Germany

3 Steinbuch Centre for Computing, KIT, Hermann-von-Helmholtz-Platz 1, 76344 Eggenstein-Leopoldshafen, Germany

4 Engler-Bunte-Institute, Division of Combustion Technology, KIT, Hermann-von-Helmholtz-Platz 1, 76344 Eggenstein-Leopoldshafen, Germany

5 Reactive Flows and Diagnostics, Technical University of Darmstadt, Otto-Berndt-Str. 3, 64287 Darmstadt, Germany 


\section{Introduction}

In the context of global warming and limited resources, the development of low-emission and high-efficiency combustion applications arises. Additionally, for the transformation to a $\mathrm{CO}_{2}$-neutral energy system, it is essential to find replacements for fossil fuels like diesel or gasoline. These alternative fuels are produced from biomass or by using $\mathrm{H}_{2}$ from renewable electricity and $\mathrm{CO}_{2}$ from the atmosphere and thereby allow for a $\mathrm{CO}_{2}$-neutral combustion process. Dimethyl ether (DME) is a promising low-emission and environmentallyfriendly alternative fuel (Semelsberger et al. 2006) and possesses the potential to become an important fuel in the future since it can be produced from biomass (Fleisch et al. 2012) or $\mathrm{H}_{2}$ (Matzen and Demirel 2016). Furthermore, for more complex flame configurations, like flame-wall interactions, DME can serve as a starting point for systematic studies of more complex oxygenated fuels.

To optimize current combustors for alternative fuels, a more profound understanding of the main combustion characteristics and especially flame-wall interactions is necessary. Thereby, numerical simulations play an important role. The description of a combustion process in a numerical simulation, however, requires the accurate modeling of the combustion chemistry. The detailed chemistry (DC) simulation solves the conservation equations of all combustion-related species directly. This, however, includes solving a large set of equations and requires a high spatial resolution which restricts the method to generic cases and simple geometries. To simulate technically relevant combustion configurations it is essential to reduce the combustion chemistry. Reduced chemistry approaches based on tabulated manifolds combine the high prediction accuracy of the chemical state of a DC simulation with low computational costs. Due to the numerous benefits of this method, several different approaches exist including FGM (van Oijen and de Goey 2000), FPI (Gicquel et al. 2000), ILDM (Maas and Pope 1992) and REDIM (Bykov and Maas 2007). The methods are based on the pre-calculation of a thermo-chemical state which is stored in a table that is accessed by control parameters. During the simulation, only the transport equations of these control parameters need to be solved to account for the chemical reaction.

Technical combustion systems are enclosed by (cold) walls, hence, the investigation of flame-wall interaction is a relevant research area. In the vicinity of the wall, the thermo-chemical reaction within the flame stagnates, leading to incomplete combustion which results in a lowered efficiency and the formation of pollutants (Poinsot and Veynante 2011). Therefore, flame-wall interaction effects have practical relevance for several thermo-chemical processes, e.g. in internal combustion engines and gas turbines (Dec and Tree 2001; Drake and Haworth 2007; Hyvönen et al. 2005). The importance of flame-wall interactions increases even further with the concept of downsizing in internal combustion engines (Dreizler and Böhm 2015) or lean-burn technologies in aero engines (Lazik et al. 2008).

Detailed investigations of flame-wall interaction are often carried out in generic configurations. Recently, flame-wall interactions in a side-wall quenching (SWQ) geometry were studied experimentally (Jainski et al. 2017a, b; Kosaka et al. 2019, 2018) and numerically (Ganter et al. 2017, 2018; Efimov et al. 2019) for methane-air flames. In the experimental studies of Kosaka et al. $(2018,2019)$ DME was considered for the first time. The first study (Kosaka et al. 2018) analyzed the near-wall thermo-chemical state of the flames using Coherent anti-Stokes Raman spectroscopy (CARS) for temperature and twophoton laser-induced fluorescence (LIF) of the CO molecule. In a second measurement campaign (Kosaka et al. 2019), the local heat-release rate (HRR) and its correlation with 
the flame structure of laminar and turbulent methane-air and DME-air flames in a SWQ geometry were analyzed using HRR imaging with simultaneous OH-LIF and $\mathrm{CH}_{2} \mathrm{O}-\mathrm{LIF}$ measurements. This work is the first complementary numerical investigation based on these experiments using DC simulations, as well as chemistry manifolds, namely Flameletgenerated Manifolds (FGM), Quenching Flamelet-generated Manifolds (QFM) and Reaction-Diffusion Manifolds (REDIM).

This paper is structured as follows. Section 2 describes the table generation procedure and the computation of the measurement signals. In Sect. 3, the experimental and numerical setups are outlined. Section 4 analyzes the local HRR of a stoichiometric methane-air and DME-air flame. In the first step, the DC simulations are compared to the measurement data using computed signals. Afterwards, the reduced chemistry manifolds FGM, QFM and REDIM are investigated. Finally, the near-wall thermo-chemical state of the flames is discussed in Sect. 5 and conclusions are drawn in Sect. 6.

\section{Numerical Methods and Modeling}

In this work, DC simulations are compared with approaches using reduced chemistry manifolds, specifically FGM, QFM and REDIM. The DC implementation and the reduced chemistry approaches are described below.

\subsection{Detailed Chemistry (DC)}

In the DC simulation the laminar flow field is described by the conservation equation for mass and momentum. Neglecting body forces, the equations read

$$
\begin{gathered}
\frac{\partial \rho}{\partial t}+\frac{\partial \rho u_{j}}{\partial x_{j}}=0 \\
\frac{\partial \rho u_{i}}{\partial t}+\frac{\partial}{\partial x_{j}}\left(\rho u_{i} u_{j}\right)=\frac{\partial}{\partial x_{j}}\left(\rho \nu\left(\frac{\partial u_{i}}{\partial x_{j}}+\frac{\partial u_{j}}{\partial x_{i}}\right)-\frac{2}{3} \rho \nu \frac{\partial u_{k}}{\partial x_{k}} \delta_{i j}\right)-\frac{\partial p}{\partial x_{i}},
\end{gathered}
$$

where $\rho$ is the density, $u$ the flow velocity, $p$ the pressure and $v$ the kinematic viscosity obtained through Sutherland's law (Saksena and Saxena 1963). The laminar flow is treated as incompressible using the low Mach number assumption. Besides these two equations, a transport equation for each species considered in the reaction mechanism has to be solved. Assuming unity Lewis number for all species, the balance equation reads

$$
\frac{\partial \rho Y_{k}}{\partial t}+\frac{\partial}{\partial x_{j}}\left(\rho u_{j} Y_{k}\right)=\frac{\partial}{\partial x_{j}}\left(\rho D \frac{\partial Y_{k}}{\partial x_{j}}\right)+\dot{\omega}_{k},
$$

where $Y_{k}$ and $\dot{\omega}_{k}$ are the mass fraction and the species source term for species $k$, and $D$ is the diffusion coefficient, respectively. Finally, the transport equation of the enthalpy $h$ as sum of sensible and enthalpy of formation reads

$$
\frac{\partial \rho h}{\partial t}+\frac{\partial}{\partial x_{j}}\left(\rho u_{j} h\right)=\frac{\partial}{\partial x_{j}}\left(\rho D \frac{\partial h}{\partial x_{j}}\right) .
$$


For the simulations, the GRI 3.0 mechanism (Smith et al. 1999) (53 species and 325 reactions) and the Zhao mechanism (Zhao et al. 2008) (55 species and 290 reactions) are used for the methane-air and the DME-air flame, respectively.

\subsection{Chemistry Manifolds}

Three models for the description of mixing-chemistry interaction, namely Flamelet-generated Manifolds (FGM), Quenching Flamelet-generated Manifolds (QFM) and Reaction-Diffusion Manifolds (REDIM), are considered. The manifolds are stored in so-called flamelet look-up tables (FLUTs) based on two control variables: the progress variable and the enthalpy. The latter is important since heat transfer to the wall must be accounted for. Thereby, the thermo-chemical quantities, the source term of the progress variable, and the computed signals are parameterized as a function of enthalpy $h$ and progress variable $Y_{\mathrm{c}}$

$$
\Psi=\Psi\left(h, Y_{\mathrm{c}}\right) \text {. }
$$

The progress variable $Y_{c}$ for methane and DME is chosen to be the mass fraction of $\mathrm{CO}_{2}$, which is consistent with previous studies of flame-wall interactions (Ganter et al. 2017, 2018). The construction of FGM, QFM and REDIM is described in Sects. 2.2.1, 2.2.2 and 2.2.3, respectively.

In a coupled simulation using FLUTs the flow field is described by the mass and momentum transport, see Eqs. (1) and (2), respectively. To account for the chemical processes, additional transport equations for the control variables are solved: The equation for the enthalpy is given in Eq. (4), while for the progress variable the balance equation reads

$$
\frac{\partial \rho Y_{c}}{\partial t}+\frac{\partial}{\partial x_{j}}\left(\rho u_{j} Y_{c}\right)=\frac{\partial}{\partial x_{j}}\left(\rho D \frac{\partial Y_{c}}{\partial x_{j}}\right)+\dot{\omega}_{Y_{c}} .
$$

After solving the transport equations, the progress variables can be used to access the thermo-chemical state stored in the FLUT. All other thermo-chemical quantities, the reaction rate and computed signals (see Sect. 2.3) are retrieved from the tabulated manifold. Figure 1 shows the look-up procedure during a coupled simulation using chemistry manifolds.

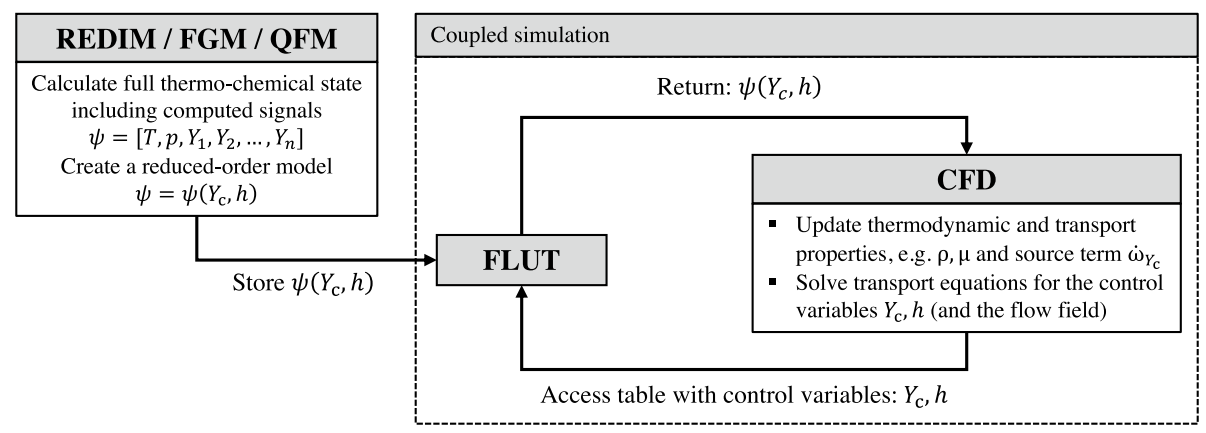

Fig. 1 Illustration of the look-up procedure of a coupled simulation using chemistry manifolds. The full thermo-chemical state is estimated by the chemistry manifold including computed signals and incorporated into a FLUT. During the coupled simulation, the transport equations of the control parameters are solved and the thermodynamic and transport properties are updated by a table look-up during runtime 


\subsubsection{Flamelet-Generated Manifolds (FGM)}

The FGM table generation procedure is detailed in van Oijen and de Goey (2000) and Ketelheun et al. (2013, 2009). The FLUT is based on a series of 1D calculations of adiabatic freely propagating flames. The corresponding simulations are performed with the in-house DC solver ULF (Universal Laminar Flame Solver) (Zschutschke et al. 2017). The wall heat transfer leads to different local enthalpy levels in the flow field that need to be considered during the flamelet generation procedure. The enthalpy variation is realized using exhaust gas recirculation. This approach has been used previously (van Oijen et al. 2016). To change the enthalpy of the specific flamelet, exhaust gases at the temperature of the unburnt gases are mixed with the fresh gas mixture. The enthalpy of the flamelet can be controlled by the ratio between fresh and burned gases. Note that the particular method to generate the different enthalpy levels has only a small influence on the resulting chemistry manifold, mostly because the flame structures do not show significant differences (Fiorina et al. 2003).

\subsubsection{Quenching Flamelet-Generated Manifolds (QFM)}

QFM are an extension of FGM which account for effects of flame quenching during flamewall interactions. In contrast to FGM, QFM are based on a transient Head-On Quenching (HOQ) flame, where the flame front of a premixed laminar flame (here methane-air or DME-air) propagates perpendicular to the wall and extinguishes due to heat losses. Thereby, the scalar diffusion due to the enthalpy gradient is incorporated into the chemistry manifold. In this study, a 2D QFM is used that consists of a single transient HOQ simulation performed with the in-house solver ULF. The resulting HOQ solution is a function of the spatial coordinate $x$ and the time $t$ and can be interpolated onto the progress variable/ enthalpy space and stored in a two-dimensional FLUT. The tabulation approach is further described in Efimov et al. (2019).

\subsubsection{Reaction-Diffusion Manifolds (REDIM)}

REDIM are an approach for reduced kinetics that accounts for both chemical reaction and molecular diffusion (Bykov and Maas 2007). It is based on the invariance condition (Gorban and Karlin 2003), and the manifold is generated by solving the REDIM evolution equation

$$
\begin{aligned}
\frac{\partial \boldsymbol{\Psi}(\boldsymbol{\theta})}{\partial t} & =\left(I-\boldsymbol{\Psi}_{\theta}(\boldsymbol{\theta}) \boldsymbol{\Psi}_{\theta}^{+}(\boldsymbol{\theta})\right) \cdot\{\boldsymbol{F}(\boldsymbol{\Psi}(\boldsymbol{\theta})) \\
& \left.+\frac{1}{\rho}\left(\overline{\bar{D}} \boldsymbol{\Psi}_{\theta}(\boldsymbol{\theta}) \operatorname{grad} \boldsymbol{\theta}\right)_{\theta} \operatorname{grad} \boldsymbol{\theta}\right\}
\end{aligned}
$$

to a stationary state (Bykov and Maas 2007). Here, $\boldsymbol{\Psi}$ is the state vector consisting of specific enthalpy, pressure and species, $\overline{\bar{D}}$ is the transport matrix with thermal conductivity and diffusion coefficients, $I$ is the identity matrix, $\boldsymbol{\Psi}_{\theta}(\boldsymbol{\theta})$ is the matrix of partial derivatives of $\boldsymbol{\Psi}$ with respect to $\boldsymbol{\theta}$ and $\boldsymbol{\Psi}_{\theta}^{+}(\boldsymbol{\theta})$ is the Moore-Penrose pseudo-inverse of $\boldsymbol{\Psi}_{\theta}(\boldsymbol{\theta})$, see e.g. Golub and van Loan (1989). $\boldsymbol{\Psi}_{\theta}(\boldsymbol{\theta})$ spans the tangential subspace to the manifold which means that the projection operator $\left(I-\boldsymbol{\Psi}_{\theta}(\boldsymbol{\theta}) \boldsymbol{\Psi}_{\theta}^{+}(\boldsymbol{\theta})\right)$ projects the vector field consisting 
of source term and diffusion term onto the normal subspace of the manifold. Due to this projection, the convective term of the evolution equation is canceled out in the REDIM evolution equation (Bykov and Maas 2007).

Before the REDIM evolution equation is solved, an initial guess and a gradient estimation need to be specified. Both are obtained from a detailed sample solution of a HOQ flame similar to the ones used for QFM. Due to the symmetry of this model system, the model configuration can be assumed to be one-dimensional and the system is described by one spatial coordinate. This means that a one-dimensional gradient estimation is obtained and all assessed states during the transient HOQ-phenomenon are used for the generation of the REDIM. Note that the gradient estimation has only a small influence on the REDIM for FWI. This has been shown in Steinhilber et al. (2017) and Strassacker et al. (2019) where the gradient estimation has been changed drastically while the results of the reduced computations only changed slightly. Therefore, even if the REDIM is generated with the gradient estimation of a HOQ flame, it can be used for computations of the SWQ configuration (Steinhilber et al. 2017; Ganter et al. 2018; Strassacker et al. 2019).

For the parametrization of the initial guess of the manifold, the specific enthalpy as well as the species $\mathrm{CO}_{2}$ are used. Note that this specification is only important for the generation of the initial guess, not for the integration procedure of the REDIM, because it is scale invariant (Bykov and Maas 2007).

The molecular transport is modeled with equal diffusivities and unity Lewis number. It should be mentioned that this assumption is made for simplicity. The use of more detailed transport models is possible (Maas and Bykov 2011; Strassacker et al. 2018a).

At the boundaries of the REDIM, boundary conditions that allow the REDIM to evolve are applied (Neagos et al. 2017; Strassacker et al. 2018b).

The computed REDIM table contains the source terms and the transport properties that are both projected onto the tangential subspace of the manifold to solve the reduced computation in the generalized coordinates $\boldsymbol{\theta}$. To implement the reduced model equation in physical variables, the REDIM is reparametrized in physical variables and the source term as well as the diffusion coefficients are appropriately reformulated to use them in Eqs. (4) and (6). The progress variables for the look-up, in the following sections referred to as control variables to be consistent with the FGM approach, are chosen to be the progress variable $Y_{\mathrm{c}}$ and the enthalpy $h$.

\subsection{Co-simulation of Computed Signals}

For the DC simulations, as well as the reduced manifolds, the OH-LIF and $\mathrm{CH}_{2} \mathrm{O}-\mathrm{LIF}$ signals are computed based on the numerical thermo-chemical state. For the chemistry manifolds, the signals are incorporated in the FLUT as a function of progress variable and enthalpy. OH (Popp et al. 2015; Hunger et al. 2017) and $\mathrm{CH}_{2} \mathrm{O}$-LIF (Popp et al. 2015) computed signals were used previously. Within these studies a detailed description of the underlying method is given. In this section, the method is briefly introduced.

The OH-LIF signal for the $Q_{1}(6)$ transition of the $A^{2} \Sigma^{+} \leftarrow X^{2} \Pi^{+}$band is calculated for the linear LIF regime with the excited-state decay rate that is dominated by collisional quenching (Kosaka et al. 2019),

$$
S_{\mathrm{OH}-\mathrm{LIF}} \propto N_{\mathrm{OH}} f_{\mathrm{J}} \frac{A}{A+Q},
$$


with $N_{\mathrm{OH}}$ being the $\mathrm{OH}$ number density, $f_{\mathrm{J}}$ the Boltzmann fraction population of the absorbing state with rotational quantum number $J$. The spontaneous emission rate is described by $A ; Q$ is the total collisional quenching rate of the excited state, which is the sum of the quenching rates for each collisional partner and is dependent on the composition and the local temperature. The total collisional quenching rate is given by

$$
Q=N_{\text {tot }} \sum_{i} X_{i} \sigma_{i}\left(\frac{8 k_{\mathrm{B}} T}{\pi \mu_{i}}\right)^{0.5},
$$

with $N_{\text {tot }}$ being the total number density. The parameters $k_{\mathrm{B}}, X_{i}, \sigma_{i}$ and $\mu_{i}$ are the Boltzmann constant, the mole fraction, the quenching cross-section and the reduced mass of the species $i$, respectively. The quenching cross sections are obtained from Tamura et al. (1998).

The calculation of the $\mathrm{CH}_{2} \mathrm{O}$-LIF signal is very challenging since it involves excitation of overlapping transitions and complex temperature and species-dependent cross sections. The $\mathrm{CH}_{2} \mathrm{O}$-LIF signal is calculated following the methodology described by Kosaka et al. (2019), which goes back to the procedure proposed in Coriton et al. (2015) and Popp et al. (2015). The $\mathrm{CH}_{2} \mathrm{O}$-LIF signal is obtained using Eqs. (8) and (9) with an additional term accounting for the temperature dependence of the quenching cross-sections and the populations of overlapping transitions. The temperature dependence is considered using the same polynomial fit as in the experiments by Kosaka et al. (2019) that is based on spectral simulations using the simulation code AsyrotWin (Birss and Ramsay 1985; Judge and Clouthier 2001). Within the simulations, the transitions near the $339 \mathrm{~nm}$ excitation of the $\widetilde{A}^{1} A_{2} \leftarrow \widetilde{X}^{1} A_{1}$ system are considered.

\section{Experimental and Numerical Setup}

Figure 2 shows the setup used in the experimental studies by Kosaka et al. $(2018,2019)$ that are examined in this work and the numerical subdomain. A homogeneous mixture of dry air and fuel enters the experimental domain from the nozzle at ambient conditions. The Reynolds number is 5.000 based on nozzle exit conditions of the fuel-air jet. The flow passes a rod with a diameter of approximately $1 \mathrm{~mm}$, where a V-flame stabilizes. The left branch of the flame approaches the wall, where the flame is quenched. The wall temperature in the experiments is controlled at the axial position $z=48 \mathrm{~mm}$ by a bath-thermostat with thermal oil. The wall temperature considered in this work is $330 \mathrm{~K}$. The experimental setup is described in more detail in Kosaka et al. (2019) and Jainski et al. (2017).

In this work two experimental studies are considered. In the first one (Kosaka et al. 2018), Coherent anti-Stokes Raman spectroscopy (CARS) and two-photon laser-induced fluorescence (LIF) of the CO molecule are used to determine the thermo-chemical state of methane-air and DME-air flames with respect to the point-wise gas phase temperatures and $\mathrm{CO}$ concentrations. Wall-normal profiles of species concentrations are measured using this technique in order to validate CFD predictions. The second one (Kosaka et al. 2019) focuses on the characterization of the local HRR. Therefore, simultaneous formaldehyde and hydroxyl radical planar laser-induced fluorescence (PLIF) measurements are used. The calculation of the measured signals is described in Sect. 2.3 and incorporated into the numerical simulations.

Following the approach by Ganter et al. (2017, 2018), the simulation domain is reduced to a two-dimensional subdomain which covers approximately $80 \%$ of the left flame branch. Consistent with previous studies for the methane-air flame, 


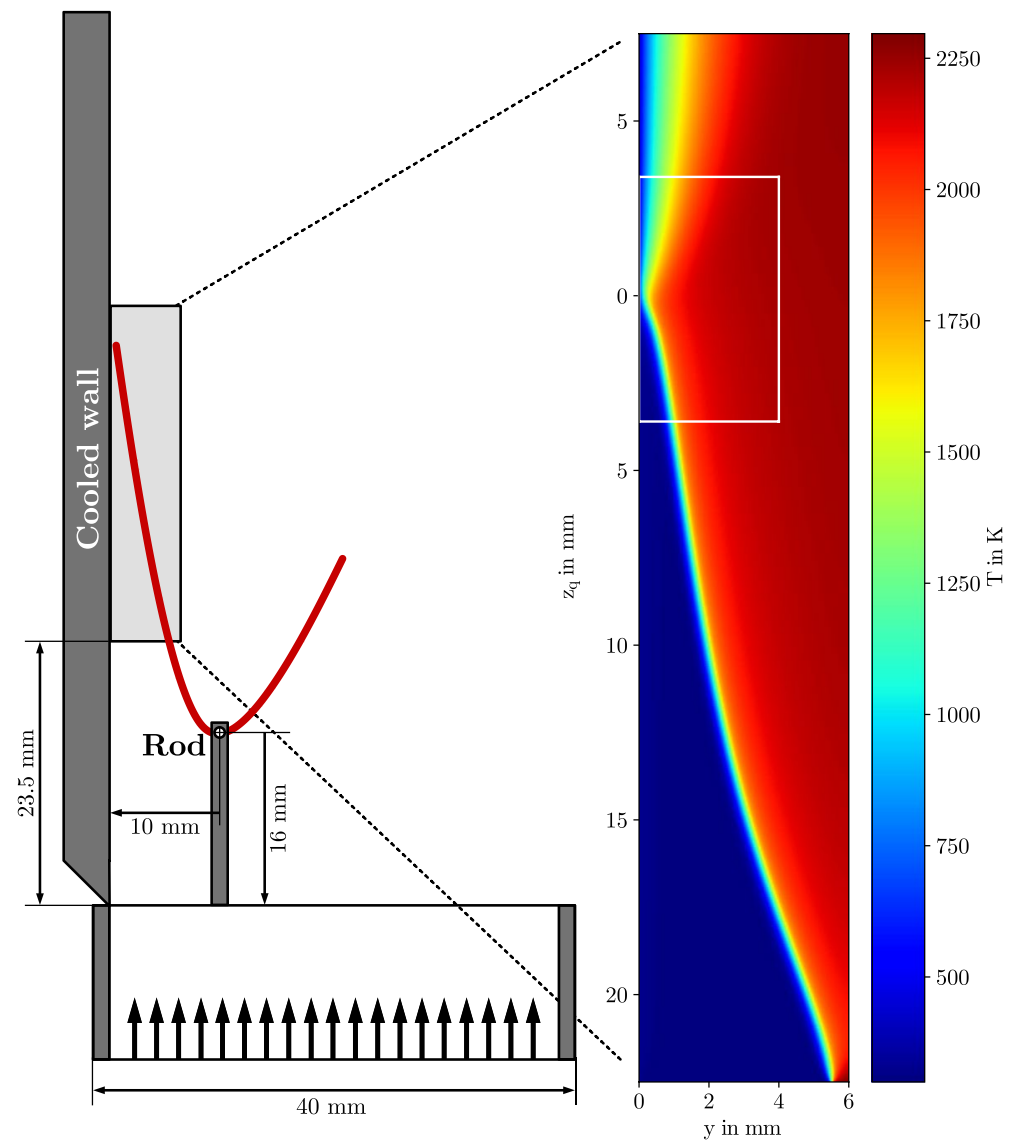

Fig. 2 Illustration of the SWQ burner geometry (left) and the numerical subdomain (right) showing the simulated temperature of the DME case. The coordinate system of the numerical domain is defined relative to the quenching height of the flame. The wall is located at $y=0$. The area within the white lines in the numerical domain marks the region of the LIF-measurements by Kosaka et al. (2019)

the computational mesh consists of a rectilinear mesh with a uniform grid size of $\Delta=50 \mu \mathrm{m}$. Due to the reduced flame thickness of the DME-air flame, the grid resolution for the DME simulations is increased towards the wall using a uniform grading. The cell size at the wall is $\Delta=24 \mu \mathrm{m}$ to ensure a sufficient resolution of the flame in the near-wall region. The computational domain is shown on the right in Fig. 2 where the DME case discussed below is displayed. The white lines depict the boundary of the domain of the LIF-measurements performed in Kosaka et al. (2019).

The numerical simulations are performed using a coupled solver using tabulated chemistry manifolds (Popp et al. 2015; Gierth et al. 2018) as well as a DC solver based on OpenFOAM. For the discretization of spatial gradients, an interpolation method from third-degree polynomials is used, while the time is discretized implicitly using the second-order backward Euler method. For all simulations, a Courant-Friedrich-Lewy number of CFL $\approx 0.1$ is employed. Since laminar flames are analyzed, the simulation is conducted until a steady solution is obtained. 
For all simulations, the wall is assumed to have a constant temperature of $330 \mathrm{~K}$. For the velocity, a no-slip condition is applied, while for the species mass fractions a zero gradient boundary condition is chosen at the wall. At the top and right outlet, zero gradient boundary conditions are applied for enthalpy, species mass fractions, and velocity. Similar to Ganter et al. $(2017,2018)$, a generic parabolic inflow velocity profile is used. The flame is stabilized by injecting hot exhaust gases at equilibrium conditions in a $0.5 \mathrm{~mm}$ wide section of the inlet. The inflow velocity of the hot exhaust gases is set to $3.81 \mathrm{~m} / \mathrm{s}$ for the methane-air flame to compensate partially for the density difference of the fresh and burned inlet gases. For the DME-air flame the velocity profile of the inlet gases is scaled according to the inflow Reynolds number. For the burned gases a constant velocity of $4.98 \mathrm{~m} / \mathrm{s}$ is used. The inlet gas mixture temperature and pressure are set to ambient conditions ( $T=300 \mathrm{~K} ; p=1 \mathrm{~atm})$. The numerical results are analyzed in a relative coordinate system, that uses the quenching point as the origin of the wall-parallel direction. The quenching height is defined based on the $\mathrm{OH}$ gradient within the flame which is described in more detail in Ganter et al. (2017).

\section{Analysis of the Local Heat-Release Rate}

In the following, the DC simulations are compared to the experimental data from Kosaka et al. $(2018,2019)$ as well as the chemistry manifolds. In this section the local HRR is analyzed as a global flame property starting with a comparison of the DC simulations and the experimental findings. Afterwards, the results obtained using chemistry manifolds are compared to corresponding DC simulations. In Sect. 5, the thermo-chemical state of the flame is analyzed with particular emphasis on the near-wall processes.

\subsection{Definition of Heat-Release Rate}

In the study by Kosaka et al. (2019), a correlation based on the product of the normalized LIF signals of $\mathrm{OH}$ and $\mathrm{CH}_{2} \mathrm{O}$ is used to predict the HRR. Based on the product of the measurement signals a normalized local HRR of the flame can be defined

$$
\mathrm{HRR}_{\text {exp }}=\frac{\left\langle S_{\mathrm{OH}-\mathrm{LIF}}\right\rangle \cdot\left\langle S_{\mathrm{CH}_{2} \mathrm{O}-\mathrm{LIF}}\right\rangle}{\max \left(\left\langle S_{\mathrm{OH}-\mathrm{LIF}}\right\rangle \cdot\left\langle S_{\mathrm{CH}_{2} \mathrm{O}-\mathrm{LIF}}\right\rangle\right)}
$$

with $\left\langle S_{\mathrm{OH}-\mathrm{LIF}}\right\rangle$ and $\left\langle S_{\mathrm{CH}_{2} \mathrm{O}-\mathrm{LIF}}\right\rangle$ being the normalized averaged measured signal intensity of $\mathrm{OH}$ and $\mathrm{CH}_{2} \mathrm{O}$, respectively. The product is normalized using the maximum value along the flame front in the lower part of the measurement domain $\left(z_{q}<-3 \mathrm{~mm}\right)$ corresponding to an unstretched flame region. To allow a direct comparison between the DC simulations and the experiments, the above HRR definition is used in both. In the DC simulation the HRR is calculated using computed signals (see Sect. 2.3). The HRR definition was validated in Kosaka et al. (2019) using three different flame configurations (1) an unstretched planar flame (2) a curved and stretched flame and (3) a HOQ configuration. For the first two configurations, the results agree qualitatively with the normalized HRR defined as

$$
\mathrm{HRR}_{\text {num }}=\frac{\sum_{i=1}^{N} \dot{\omega}_{i} h_{f, i}}{\max \left(\sum_{i=1}^{N} \dot{\omega}_{i} h_{f, i}\right)},
$$


where $\dot{\omega}_{i}$ is the source term and $h_{f, i}$ is the enthalpy of formation of the species $i$. For the unsteady HOQ configuration, the HRR correlations agree reasonably well until the point where the flame is quenched at the wall. During the quenching process the radicals in the vicinity of the wall are consumed faster than the HRR declines and the correlation deteriorates (Kosaka et al. 2019). In this work, the validation is extended to the steady SWQ scenario, which will be discussed further for the methane-air flame next.

\subsection{Comparison to Experimental Findings}

In "Appendix" the flame structure and the specifics of the methane-air and DME-air flame are discussed. Thereby, the fundamental flame characteristics of the two fuels are outlined. In the following, we focus on the local HRR during SWQ. Figure 3 shows the local HRR over the wall-normal direction $y$ and the relative quenching height $z_{q}$ for the methane case. The experimental results are displayed in subfigure (a), while (b) and (c) correspond to the DC simulation with the HRR definition based on Eqs. (10) and (11), respectively. Additionally, the white line in the Fig. $3 \mathrm{c}$ displays the isoline of $\mathrm{HRR}_{\text {num }}=0.05$. The HRR definition used in the experiments $\mathrm{HRR}_{\text {exp }}$ is in very good agreement with $\mathrm{HRR}_{\text {num }}$ considering HRR values greater than $5 \%$ (see Fig. 3b, c). At the edges of the reaction zone, however, the formaldehyde vanishes and the prediction based on $\mathrm{HRR}_{\text {exp }}$ deteriorates. These results are consistent with the HOQ scenario analyzed in Kosaka et al. (2019) and extend those findings to the more complex SWQ configuration. Similar results are obtained for the DME-air flame that are not shown here for brevity.

For a consistent comparison with the experimental data, $\mathrm{HRR}_{\exp }$ is used. The HRR prediction of the DC simulation in Fig. 3b shows an overall good agreement with the measurements shown in Fig. 3a. Nevertheless, the experimental results show a broader reaction zone. This is particularly evident in the lower part of the flame where the flame structure should correspond to an unperturbed laminar flame. Potential reasons for these differences are (1) the measurement resolution, (2) the measurement uncertainties caused by the image intensifiers, and (3) fluctuations in the instantaneous flame position and flame angle of the laminar flame due to Helmholtz resonances originating from the plenum of the burner.
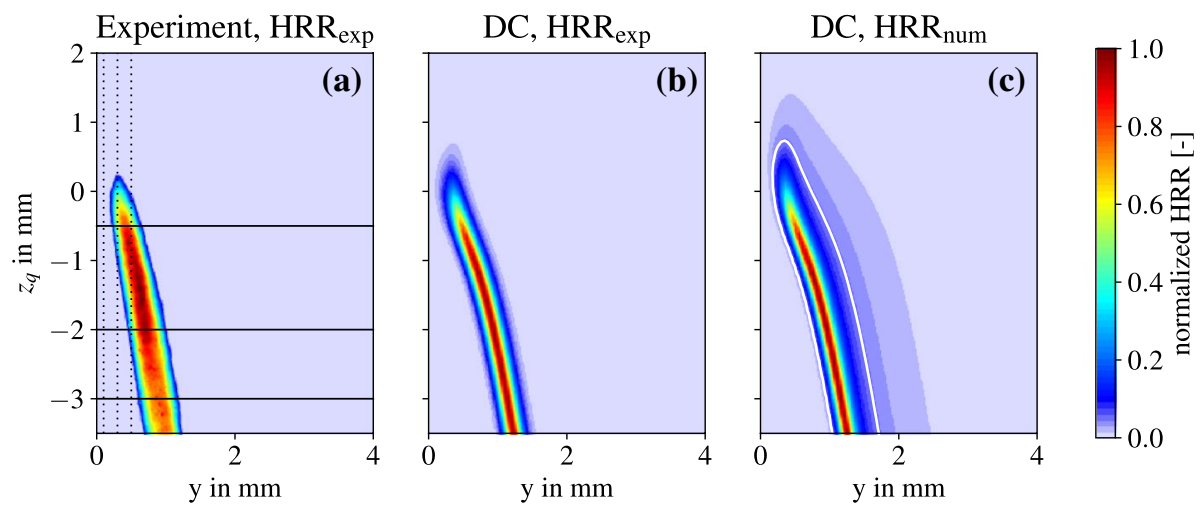

Fig. 3 Local HRR in the methane-air flame. The experimental results are taken from Kosaka et al. (2019). The HRR definitions $\mathrm{HRR}_{\text {exp }}$ and $\mathrm{HRR}_{\text {num }}$ correspond to Eqs. (10) and (11), respectively. The white isoline in $\mathbf{c}$ shows a HRR value of $5 \%$ of the maximum HRR. The black solid lines in a display the wall-normal lines along which the HRR profiles are extracted. The thermo-chemical state that is discussed in Sect. 5 is analyzed along the black dotted lines 
Even though these fluctuations are accounted for in the post-processing of the measurements by shifting the individual images vertically on the average quenching point, uncertainties regarding the exact flame position and flame angle remain (Jainski et al. 2017a, b; Kosaka et al. 2018, 2019).

To further investigate potential reasons for the differences in the numerical simulation and the experimental findings, the HRR based on Eq. (10) is analyzed along wall-normal profiles that are shown as black solid lines in Fig. 3a. The obtained HRR profiles are normalized such that the integral of the numerical simulations and the experimental data match. This allows a comparison of the shape of the HRR distribution along the wall normal lines. Then, artificial measurement uncertainties are superimposed onto the simulation results using a box filter with a variable filter width $\Delta$. Since the measurement uncertainties cannot be specified, the use of a box filter presents a worst-case estimation of errors originating from the measurement uncertainties. Figure 4 displays the corresponding profiles of the methane case in a coordinate system relative to the HRR peak. While in the undisturbed part of the flame the HRR peak position differs by over $200 \mu \mathrm{m}$, in the near-wall region the shift vanishes. Therefore, the thermo-chemical state during quenching is unaffected by this shift.

The filtering of the signals leads to a broadening of the HRR profile in the $y$-direction. In the unperturbed part of the flame $\left(z_{q}=-3 \mathrm{~mm}\right)$, a filter width of $\Delta>300 \mu \mathrm{m}$ is necessary to match the width of the experimental profile. With decreasing distance from the quenching height, however, the required filter width decreases. In the vicinity of the quenching height $\left(z_{q}=-0.5 \mathrm{~mm}\right)$, a filter with of $\Delta=200 \mu \mathrm{m}$ is sufficient. Assuming remaining uncertainties in the flame angle in the post-processed measurement results, the measurement uncertainty would increase with increasing distance from the quenching point, since the spatial distortion increases proportionally with the distance. This leads to an increasing filter width with increasing distance to the quenching height.

Figure 5 shows the corresponding HRR profiles of the DME case. In contrast to the methane-air flame, the DC simulation of the DME case predicts the thickness of the HRR profile in the undisturbed part of the flame satisfactorily using a filter width of $\Delta \approx 250 \mu \mathrm{m}$. Additionally, the position of the HRR peak in the numerical simulations of the DME-air flame is shifted between $60 \mu \mathrm{m}$ and $140 \mu \mathrm{m}$ to larger wall distances over the whole flame
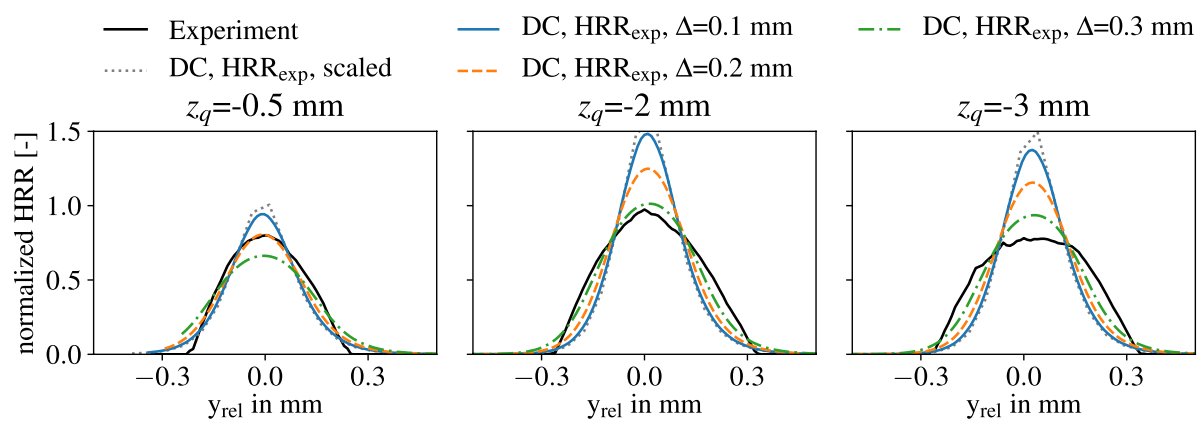

Fig. 4 Local HRR profiles of the methane-air flame based on $H_{R R}{ }_{\exp }$ [see Eq. (10)]. The experimental results are taken from Kosaka et al. (2019). The profiles are extracted along wall-normal lines at different heights $z_{q}$ that are displayed as black solid lines in Fig. 3a. The extracted profiles are scaled to match the integral of the experimental signal. The obtained signal is filtered using a box filter with variable filter widths $\Delta$. The numerical profiles are shifted towards the wall by $\Delta_{y}=50 \mu \mathrm{m}, 225 \mu \mathrm{m}$ and $250 \mu \mathrm{m}$ for $z_{q}=-0.5 \mathrm{~mm},-2 \mathrm{~mm}$ and $-3 \mathrm{~mm}$, respectively 

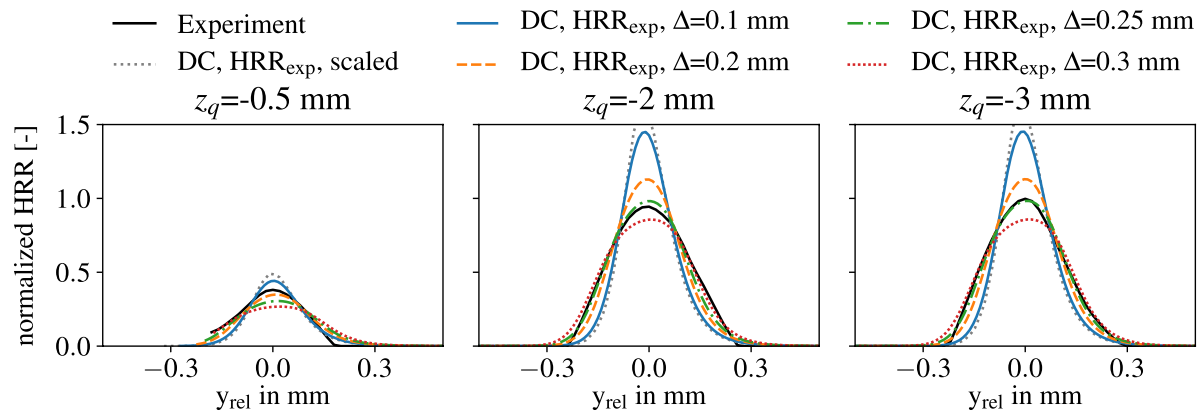

Fig. 5 Local HRR profiles of the DME-air flame based on HRR ${ }_{\text {exp }}$ [see Eq. (10)]. The experimental results are taken from Kosaka et al. (2019). The profiles are extracted along wall-normal lines at different heights $z_{q}$ that are displayed as black solid lines in Fig. 3a. The extracted profiles are scaled to match the integral of the experimental signal. The obtained signal is filtered using a box filter with variable filter widths $\Delta$. The numerical profiles are shifted towards the wall by $\Delta_{y}=140 \mu \mathrm{m}, 115 \mu \mathrm{m}$ and $60 \mu \mathrm{m}$ for $z_{q}=-0.5 \mathrm{~mm}$, $-2 \mathrm{~mm}$ and $-3 \mathrm{~mm}$, respectively

front. Other than in the methane case, the shift does not decrease in the near-wall region $\left(\Delta_{y}=140 \mu \mathrm{m}\right)$. The thermo-chemical state that is discussed in Sect. 5 shows a similar shift. Even though great care has been taken to determine the exact distance to the wall in the experiments, thermal disturbances and beam steering, that affect the determination of the horizontal position of the wall, cannot be avoided. The resulting uncertainties lie in the range of the deviations.

Compared to the methane-air flame (Fig. 4), the DME-air flame shows a thinner HRR zone in the undisturbed part of the flame. This is related to the flame thickness of a stochiometric DME-air flame compared to a methane-air flame and can already be observed in a simple freely propagating flame, see "Appendix" for further detail. During quenching, the HRR peak decreases, while the width of the HRR zone is unaffected by the quenching process. It is interesting to note that the different flame thickness of the methane-air and DME-air flame during quenching can be explained by fuel characteristics that can already be observed in very simple flame configurations, like a freely propagating flame.

Finally, Fig. 6a, b display the 2D profiles of the unfiltered and filtered HRR using a filter width of $\Delta=300 \mu \mathrm{m}$ and $\Delta=250 \mu \mathrm{m}$ for the methane-air and DME-air flame, respectively. The filtered and unfiltered signals agree reasonably well with the measurement data. Based on the discussion above, filtered signals result in a thickened HRR profile in the undisturbed part of the flame which better reflect the measurement results. This finding, however, does not imply that the measurement resolution is only $\Delta$, rather we believe that the potential reasons (1)-(3) are responsible for the experimentally observed broadening. As mentioned above, the agreement between the experiment and simulation deteriorates with increasing distance from the quenching height for the methane case. This could be related to the fluctuations in the instantaneous flame position.

\subsection{Prediction of the Heat-Release Rate Using Tabulated Manifold Approaches}

While in the previous section DC simulations for the HRR were compared to experimental data, in the following the suitability of manifold-based approaches to predict the near-wall HRR, is investigated. Therefore, the manifold-based approaches are compared to their corresponding DC simulations. The HRR definition used for the comparison is based on Eq. (11). 

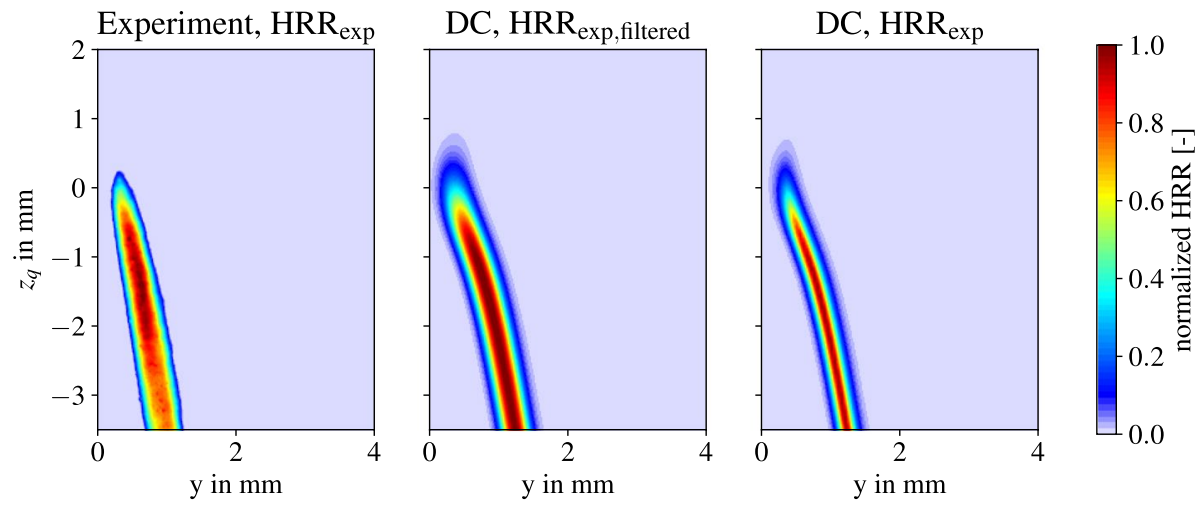

(a) Methane-air flame, $\Delta=300 \mu \mathrm{m}$
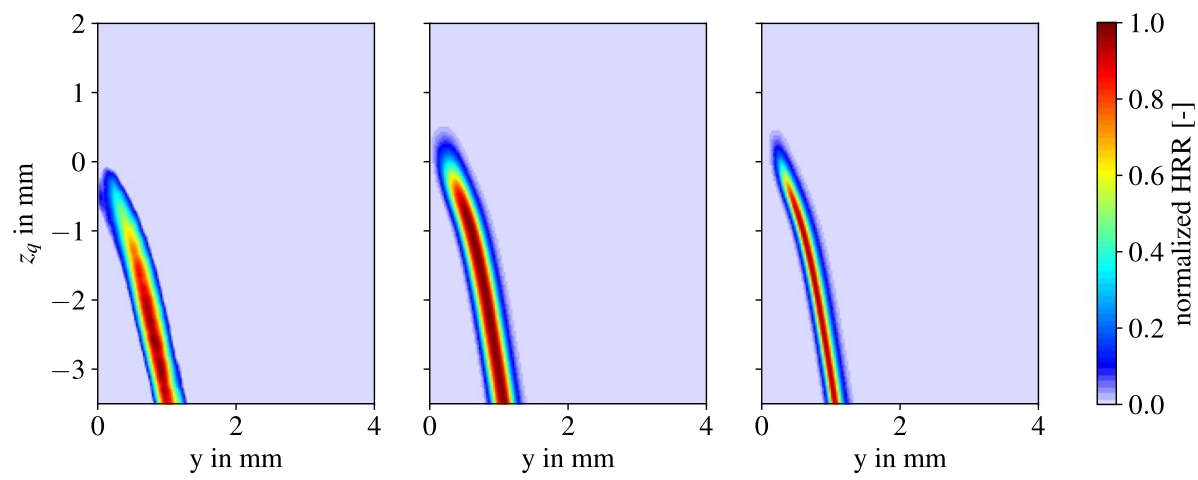

(b) DME-air flame, $\Delta=250 \mu \mathrm{m}$

Fig. 6 2D plot of the local HRR based on $\mathrm{HRR}_{\text {exp }}$ [see Eq. (10)]. In the figure, the experimental results (left) and two versions of the DC simulations, filtered (middle) using a box filter with the filter width $\Delta$ and unfiltered (right) are shown. The experimental results are taken from Kosaka et al. (2019)

Figure 7 displays the obtained results for the methane-air (top) and DME-air (bottom) flame along wall-normal lines. For both fuels, FGM, QFM and REDIM compare favorably with the DC results. This is not a trivial finding, since within the near-wall region the enthalpy level in the flame varies due to heat transfer to the wall. These different enthalpy levels in the flame are considered in the manifolds by using the enthalpy as a control variable of the FLUT. The agreement of the manifolds with the DC simulations reveals, that the manifolds, even though they use different approaches to obtain the thermo-chemical state, are suitable to predict the HRR in the near-wall region and thereby to account for the enthalpy losses to the wall. This is consistent with previous findings that showed a good prediction quality for the tabulation strategies regarding global flame properties in methane-air flames (Ganter et al. 2017, 2018; Efimov et al. 2019). 


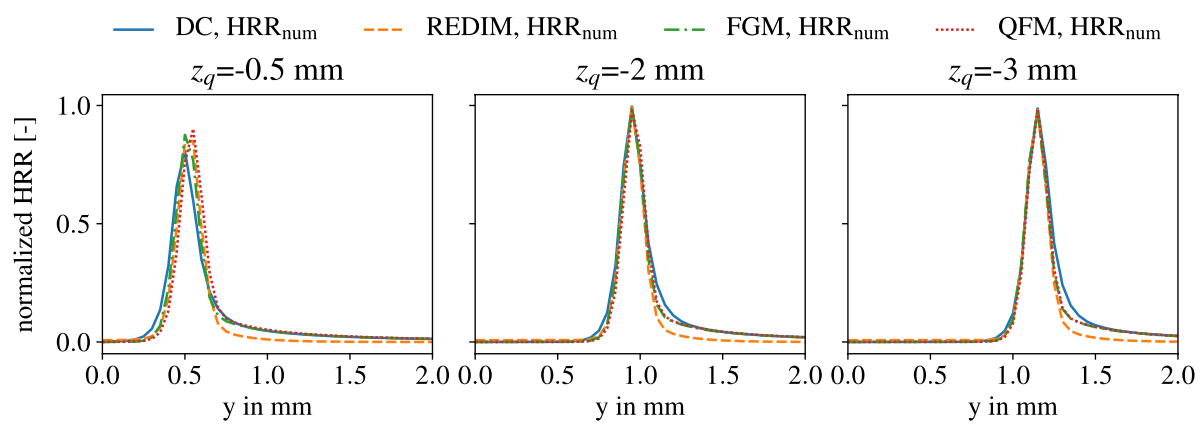

(a) Methane-air flame
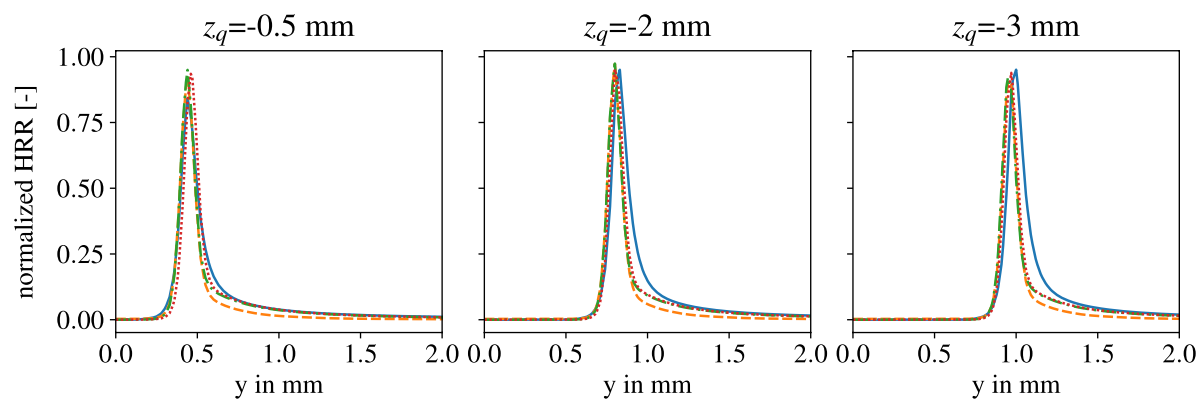

(b) DME-air flame

Fig. 7 Local HRR profiles based on the HRR definition $H_{R R}$ num [see Eq. (11)] for the DC, REDIM, FGM and QFM simulation. The profiles are extracted along wall-normal lines at different heights $z_{q}$ that are displayed as black solid lines in Fig. 3a

\section{Analysis of the Thermo-Chemical State}

In this section, the thermo-chemical state of the flames is analyzed. The thermo-chemical state, specifically looking at CO and temperature, of methane-air flames in the SWQ burner was discussed previously in various studies (Ganter et al. 2017, 2018; Efimov et al. 2019). Thereby laminar flames and different tabulation strategies were considered. This is the first study for DME-air flames in the SWQ configuration.

\subsection{Comparison to Experimental Findings}

The numerical simulations of the methane-air flame conducted here, confirm the observations published in the previous studies and, therefore, are not shown for brevity. In the following we focus on the DC simulation of the DME-air flame by first comparing with the available experimental data. Afterwards, the suitability of the chemistry manifolds to describe the near-wall thermo-chemical state is analyzed. The analysis is performed using FGM, QFM, an extension of FGM, and REDIM. The latter two have been specifically designed to model the thermo-chemical state during flame-wall interactions (Strassacker et al. 2018a, b; Efimov et al. 2019). Note, as stated above, REDIM are not very sensitive with respect to the gradient estimation. 


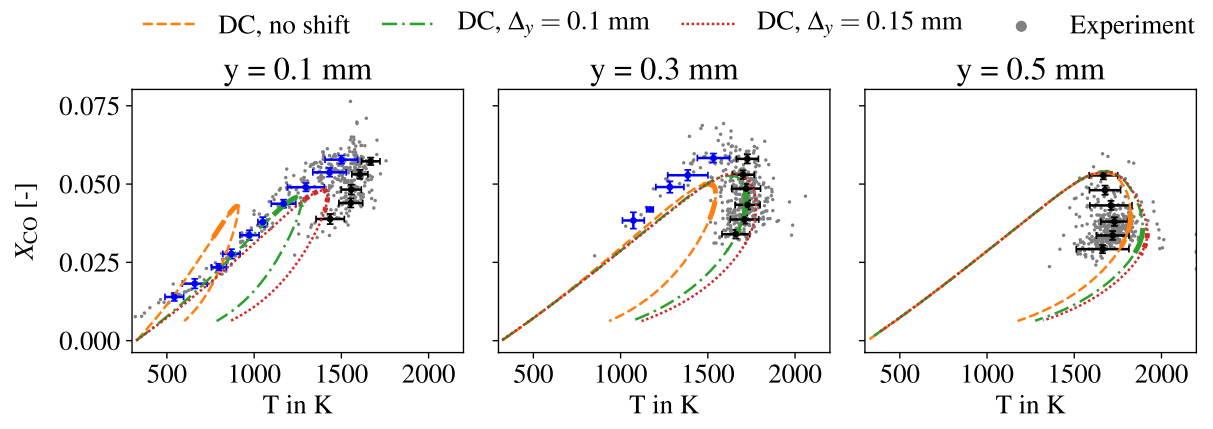

Fig. 8 Thermo-chemical state of the DME-air flame at different axial positions. The measurement data previously reported in Kosaka et al. (2018) is shown as scatter together with the conditional mean and the standard deviation separated into a CO formation (blue) and oxidation branch (black). In addition, the DC simulation results are plotted along wall-parallel lines for different axial shifts $\Delta_{y}$. The thick part of the DC lines corresponds to an area $\pm 200 \mu \mathrm{m}$ around the quenching height. The wall-parallel lines are displayed as dotted black lines in Fig. 3a. Here, the shifted wall-parallel lines are not displayed

In the experimental study by Kosaka et al. (2018) (see also Sect. 3) the thermochemical state of the flame was characterized using the $\mathrm{CO}$ concentration from $\mathrm{CO}$ LIF measurements and the temperature based on CARS. Figure 8 shows the thermochemical state for the DME-air flame at wall distances of $y=0.1 \mathrm{~mm}, y=0.3 \mathrm{~mm}$ and $y=0.5 \mathrm{~mm}$ along a wall-normal line at quenching height. The experimental data is shown as scatter. The conditional mean as well as the standard deviation is included, separated into a $\mathrm{CO}$ formation (blue dots) and a $\mathrm{CO}$ oxidation (black dots) branch, based on the CO-T probe volume position. As mentioned previously, the flame tip is not stationary in the experiments, but it fluctuates around the quenching point reported to be in the range of $\pm 200 \mu \mathrm{m}$ (Kosaka et al. 2018). These flame tip fluctuations are not present in the DC simulations. To allow a comparison with the experimental data, the CO-T state of the DC simulation is plotted along wall-parallel lines. Thereby, the flame fluctuations are captured, since both, the $\mathrm{CO}$ formation, as well as the oxidation branch, are crossed. The thick solid part of the lines corresponds to the area $\pm 200 \mu \mathrm{m}$ around the quenching height. Even though the majority of the points lies within this range, it seems that the measurement uncertainty of the flame tip position is higher than anticipated in the near-wall region ( $y=0.1 \mathrm{~mm}$ and $y=0.3 \mathrm{~mm}$ ). Similar observations were made in a recent numerical study of the SWQ burner (Zirwes et al. 2019). Therefore, the numerical results are plotted also over the entire simulation domain.

Additionally, as discussed in the previous section, the analysis of the local HRR reveals a deviation between the measurement data and the simulations results regarding the wall-normal position of the HRR peak of around $140 \mu \mathrm{m}$. This difference in the flame position effects the thermo-chemical state of the flame. To asses the sensitivity of the wall-normal flame distance to the wall on the thermo-chemical state, the extraction lines of the DC simulations are shifted in wall-normal direction by $100 \mu \mathrm{m}$ and $150 \mu \mathrm{m}$. While the region far away from the wall $(y=0.5 \mathrm{~mm})$ shows a small sensitivity, the near-wall region is strongly affected by uncertainties in the wall position. This demonstrates the high complexity of the near-wall diagnostics, since on the one hand, the thermo-chemical state is very sensitive to the wall-normal position, on the other hand, the measurements very close to the wall are especially challenging. After correcting for 

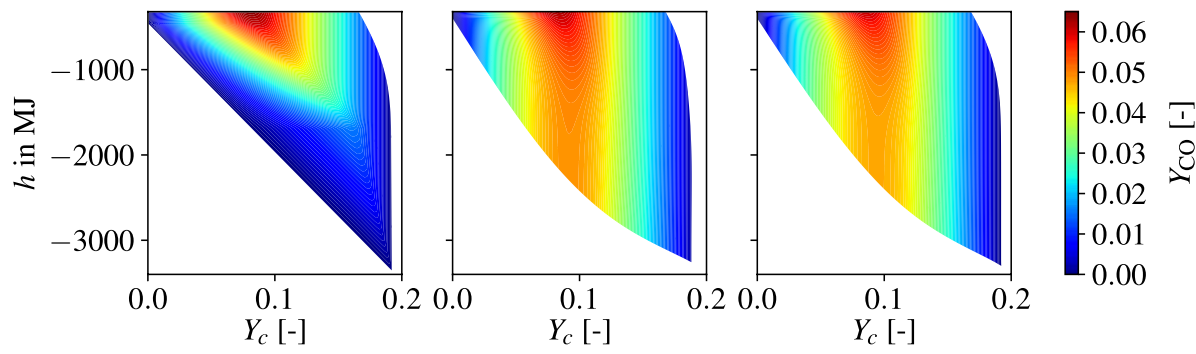

Fig. 9 Mass fraction of CO for FGM (left), QFM (middle) and REDIM (right) shown in the enthalpy progress variable space
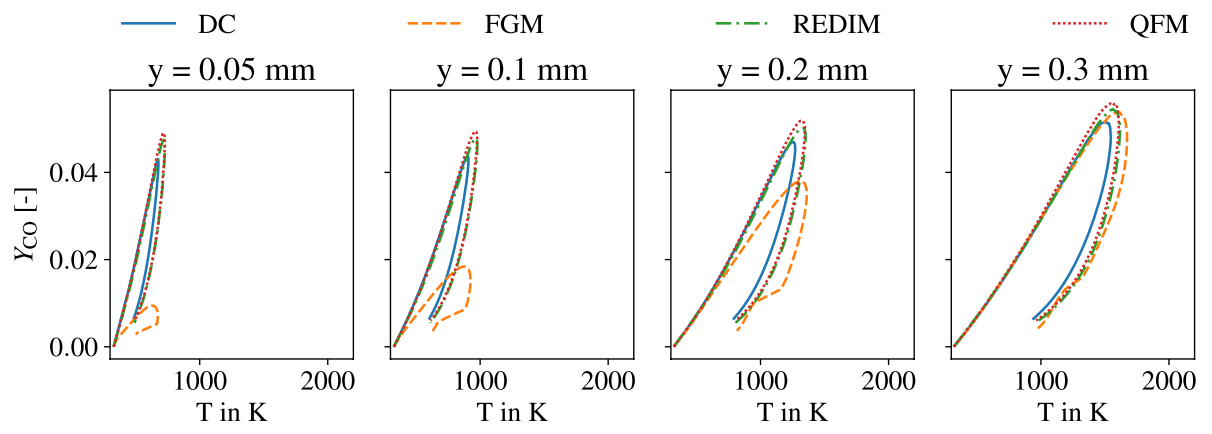

Fig. 10 Thermo-chemical state of the DME-air flame for the DC simulation, REDIM and FGM at different axial positions. The extracted lines are parallel to the black dotted lines displayed in Fig $3 \mathrm{a}$

the observed shift, the DC results achieve good agreement with the experimental data in the near-wall region ( $y=0.1 \mathrm{~mm}$ and $y=0.3 \mathrm{~mm}$ ).

\subsection{Prediction of the Thermo-Chemical State Using Tabulated Manifold Approaches}

In the following, near-wall $\mathrm{CO}$ predictions for the reduced chemistry manifolds, namely FGM, QFM and REDIM, are compared and analyzed. Thereby, the DC simulations serve as a reference solution. While FGM are based on a series of freely propagating flames with different enthalpy levels, QFM and REDIM are based on a transient 1D HOQ simulation, see Sect. 2. Diffusive effects in enthalpy direction that are not captured in the FGM tabulation can be accounted for. Figure 9 shows the $\mathrm{CO}$ mass fraction in the enthalpy progress variable space for the chemistry manifolds. While QFM and REDIM do not show significant differences, FGM predict a lower CO concentration in the near-wall region (left boundary). It is interesting to note that QFM as well as REDIM lead to a similar thermo-chemical state, even though the table generation procedure differs between the two methods.

In Fig. 10, the tabulated chemistry simulations are compared with the DC simulation results for different wall distances. In the undisturbed region of the flame $(y>0.3 \mathrm{~mm})$ the FGM prediction is of comparable accuracy to REDIM and QFM. All manifolds over-predict the $\mathrm{CO}$ concentration of the flame slightly. In the near-wall region, however, the FGM prediction, deteriorates with decreasing wall-normal distance. This is caused by diffusive 
effects in enthalpy direction that are not captured by the FGM creation procedure described in Sect. 2.2.1. Similar observations were made for methane-air flames in previous studies (Strassacker et al. 2018b; Ganter et al. 2017, 2018; Efimov et al. 2019). As mentioned above, QFM and REDIM are able to capture these effects and, hence, show a significantly improved prediction of the $\mathrm{CO}$ concentration at the wall. While, the peak $\mathrm{CO}$ concentration is still over-predicted. This is evident in the near-wall region and the undisturbed part of the flame. Efimov et al. (2019) showed (for methane-air) that even though the species diffusion in the direction of the enthalpy gradient is included in an HOQ configuration, its rate might be over-predicted leading to an increased $\mathrm{CO}$ concentration in the vicinity of the wall. This is caused by the rate of heat loss to the wall which is up to a factor of two lower in the SWQ scenario compared to HOQ. To model these effects, a third control variable accounting for the varying heat transfer rate to the wall could be introduced in future REDIM tabulations or QFM (Efimov et al. 2019).

\section{Conclusion}

This work is the first comparison of experimental and numerical data of local HRR imaging in an SWQ configuration of methane-air and DME-air flames considering DC simulations and reduced chemistry simulations using FGM, QFM and REDIM. Additionally, the thermo-chemical state during quenching of a DME-air flame is analyzed with respect to the prediction of carbon monoxide and temperature in the near-wall region. Previously, this was only performed for other fuels, especially methane. The main conclusions are as follows:

1. The HRR definition based on the normalized product of OH-LIF and $\mathrm{CH}_{2} \mathrm{O}-\mathrm{LIF}$ signals used in the measurements shows very good agreement with a HRR definition based on the species source term and the enthalpy of formation for HRR $>5 \%$ in the SWQ configuration.

2. The experimental HRR results are compared to DC simulations using computed signals. Measurement uncertainties are superimposed on the numerical simulation which results in a favorable comparison with the experiments.

3. The normalized HRR prediction using reduced chemistry manifolds, namely FGM, QFM and REDIM, are in very good agreement with the DC simulations.

4. The thermo-chemical state shows a high sensitivity towards the wall-normal position of the flame in the near-wall region.

5. In the numerical simulations, the DME-air flame burns further away from the wall. This shift is observed in the measurement of the HRR profiles, as well as in the thermochemical state of the flame. Correcting for the shift, the thermo-chemical states of the DC simulations show satisfactory agreement with the experimental results.

6. FGM show a systematic prediction deficiency of the near-wall CO concentration, while REDIM and QFM are able to capture the thermo-chemical state more accurately. REDIM and QFM do not show a significant difference regarding the CO prediction capability. This extends previous findings for methane-air flames. This is the first direct comparison of QFM and REDIM.

Overall, the combined analysis of experimental and numerical data for the HRR using computed signals allows for a direct comparison with the measurements, including 
measurement uncertainties. The analysis of different chemistry manifolds shows consistent trends with previous studies using methane-air flames, however, these findings have now been extended to more complex oxygenated fuels such as DME.

Acknowledgements Open Access funding provided by Projekt DEAL. Funded by the Deutsche Forschungsgemeinschaft (DFG, German Research Foundation)—Projektnummer 237267381—TRR 150

\section{Compliance with Ethical Standards}

Conflicts of interest The authors declare that they have no conflict of interest.

Open Access This article is licensed under a Creative Commons Attribution 4.0 International License, which permits use, sharing, adaptation, distribution and reproduction in any medium or format, as long as you give appropriate credit to the original author(s) and the source, provide a link to the Creative Commons licence, and indicate if changes were made. The images or other third party material in this article are included in the article's Creative Commons licence, unless indicated otherwise in a credit line to the material. If material is not included in the article's Creative Commons licence and your intended use is not permitted by statutory regulation or exceeds the permitted use, you will need to obtain permission directly from the copyright holder. To view a copy of this licence, visit http://creativecommons.org/licenses/by/4.0/.

\section{Appendix: Flame Structure of Freely Propagating Methane-Air and DME-Air Flames}

Figure 11 displays the thermo-chemical structure of a freely propagating methaneair (left) and DME-air (right) flame under stoichiometric conditions calculated under unity Lewis number assumption. The DME-air flame has a higher burning velocity and a decreased flame thickness. Using the definition of the thermal flame thickness based on the temperature gradient in Poinsot and Veynante (2011), the flame thickness of the displayed methane-air and DME-air flame are $0.5 \mathrm{~mm}$ and $0.4 \mathrm{~mm}$, respectively. The DME-air flame has an overall increased fuel and $\mathrm{CO}_{2}$ mass fraction and shows steeper gradients. A similar observation can be made for $\mathrm{CO}$ and $\mathrm{OH}$. As a result of the more complex fuel structure, the combustion of DME produces a higher amount of

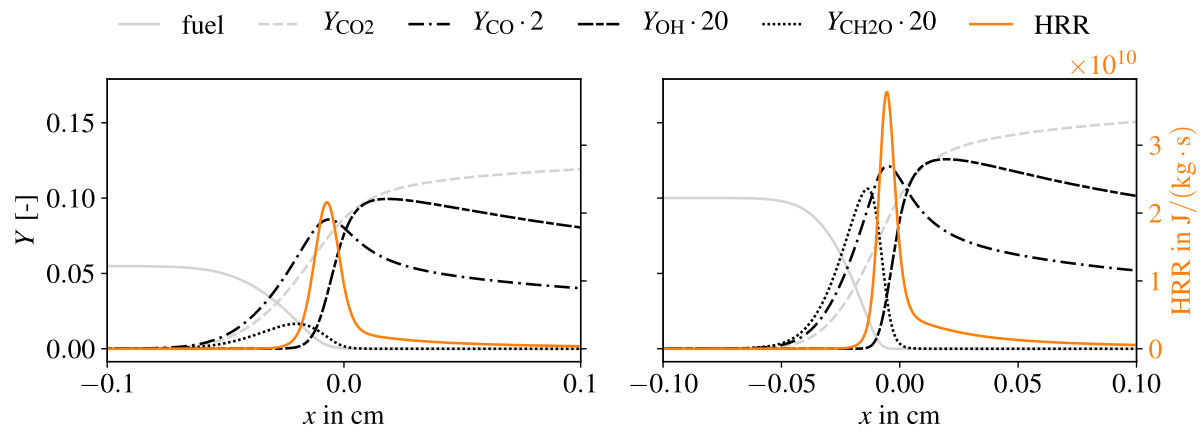

Fig. 11 Flame structure of a freely propagating methane-air (left) and DME-air (right) flame. The species mass fractions of major species are shown in grey, minor species in black and the local HRR of the flame is shown in orange. The mass fraction of $\mathrm{CO}$ is multiplied by a factor of 2 , while $\mathrm{CH}_{2} \mathrm{O}$ and $\mathrm{OH}$ are multiplied by 20 
hydrocarbons, which is apparent in the amount of formaldehyde that is by a factor of 6 higher in the DME-air flame.

In addition to the species mass fractions, the local HRR defined as HRR $=-\sum_{i=1}^{N} \dot{\omega}_{i} h_{f, i}$ is shown in Fig. 11. The DME-air flame shows a higher local HRR rate and steeper gradients compared to the methane-air flame. Similar observations can be made in the SWQ configuration, where the DME-air flame has a thinner and steeper HRR zone in the undisturbed part of the flame, see e.g. Figs. 4 and 5 on the right. The characteristics in the unperturbed part of the flame can also be observed in the near-wall region of the two flames.

\section{References}

Birss, F., Ramsay, D.: Computer assistance in the analysis of molecular spectra: I. Rotational structure of high resolution singlet-singlet bands. Comput. Phys. Commun. 38(1), 83-112 (1985)

Bykov, V., Maas, U.: The extension of the ILDM concept to reaction-diffusion manifolds. Combust. Theor. Model. 11(6), 839-862 (2007)

Coriton, B., Zendehdel, M., Ukai, S., Kronenburg, A., Stein, O.T., Im, S.K., Gamba, M., Frank, J.H.: Imaging measurements and LES-CMC modeling of a partially-premixed turbulent dimethyl ether/ air jet flame. Proc. Combust. Inst. 35, 1251-1258 (2015)

Dec, J.E., Tree, D.R.: Diffusion-flame/wall interactions in a heavy-duty DI diesel engine. SAE Trans. 110, 1599-1617 (2001)

Drake, M.C., Haworth, D.C.: Advanced gasoline engine development using optical diagnostics and numerical modeling. Proc. Combust. Inst. 31, 99-124 (2007)

Dreizler, A., Böhm, B.: Advanced laser diagnostics for an improved understanding of premixed flamewall interactions. Proc. Combust. Inst. 35, 37-64 (2015)

Efimov, D.V., de Goey, P., van Oijen, J.A.: QFM: quenching flamelet-generated manifold for modelling flame-wall interactions. Combust. Theory Model. 24, 72-104 (2019)

Fiorina, B., Baron, R., Gicquel, O., Thevenin, D., Carpentier, S., Darabiha, N.: Modelling non-adiabatic partially premixed flames using flame-prolongation of ILDM. Combust. Theor. Model. 7(3), 449470 (2003)

Fleisch, T.H., Basu, A., Sills, R.A.: Introduction and advancement of a new clean global fuel: the status of DME developments in China and beyond. J. Nat. Gas Sci. Eng. 9, 94-107 (2012)

Ganter, S., Heinrich, A., Meier, T., Kuenne, G., Jainski, C., Rißmann, M.C., Dreizler, A., Janicka, J.: Numerical analysis of laminar methane-air side-wall-quenching. Combust. Flame 186, 299-310 (2017)

Ganter, S., Straßacker, C., Kuenne, G., Meier, T., Heinrich, A., Maas, U., Janicka, J.: Laminar near-wall combustion: analysis of tabulated chemistry simulations by means of detailed kinetics. Int. J. Heat Fluid Flow 70, 259-270 (2018)

Gicquel, O., Darabiha, N., Thévenin, D.: Laminar premixed hydrogen/air counterflow flame simulations using flame prolongation of ILDM with differential diffusion. Proc. Combust. Inst. 28(2), 19011908 (2000)

Gierth, S., Hunger, F., Popp, S., Wu, H., Ihme, M., Hasse, C.: Assessment of differential diffusion effects in flamelet modeling of oxy-fuel flames. Combust. Flame 197, 134-144 (2018)

Golub, G.H., van Loan, C.F.: Matrix Computation. Technical report, Baltimore (1989)

Gorban, A.N., Karlin, I.V.: Method of invariant manifold for chemical kinetics. Chem. Eng. Sci. 58(21), 4751-4768 (2003)

Hunger, F., Zulkifli, M.F., Williams, B.A., Beyrau, F., Hasse, C.: Comparative flame structure investigation of normal and inverse turbulent non-premixed oxy-fuel flames using experimentally recorded and numerically predicted Rayleigh and OH-PLIF signals. Proc. Combust. Inst. 36(2), 1713-1720 (2017)

Hyvönen, J., Haraldsson, G., Johansson, B.: Operating Conditions Using Spark Assisted HCCI Combustion During Combustion Mode Transfer to SI in a Multi-cylinder VCR-HCCI Engine. SAE Technical Paper (2005)

Jainski, C., Rißmann, M., Böhm, B., Dreizler, A.: Experimental investigation of flame surface density and mean reaction rate during flame-wall interaction. Proc. Combust. Inst. 36(2), 1827-1834 (2017a)

Jainski, C., Rißmann, M., Böhm, B., Janicka, J., Dreizler, A.: Sidewall quenching of atmospheric laminar premixed flames studied by laser-based diagnostics. Combust. Flame 183, 271-282 (2017b) 
Judge, R., Clouthier, D.J.: AsyrotWin: a 32-bit Windows version of Asyrot, A program for the analysis of high resolution singlet-singlet band spectra of asymmetric tops. Comput. Phys. Commun. 135(3), 293-311 (2001)

Ketelheun, A., Kuenne, G., Janicka, J.: Heat transfer modeling in the context of large eddy simulation of premixed combustion with tabulated chemistry. Flow Turbul. Combust. 91(4), 867-893 (2013)

Ketelheun, A., Olbricht, C., Hahn, F., Janicka, J.: Premixed Generated Manifolds for the Computation of Technical Combustion Systems. Volume 2: Combustion, Fuels and Emissions. Turbo Expo: Power for Land, Sea, and Air, pp. 695-705 (2009)

Kosaka, H., Zentgraf, F., Scholtissek, A., Bischoff, L., Häber, T., Suntz, R., Albert, B., Hasse, C., Dreizler, A.: Wall heat fluxes and CO formation/oxidation during laminar and turbulent side-wall quenching of methane and DME flames. Int. J. Heat Fluid Flow 70(January), 181-192 (2018)

Kosaka, H., Zentgraf, F., Scholtissek, A., Hasse, C., Dreizler, A.: Effect of flame-wall interaction on local heat release of methane and DME combustion in a side-wall quenching geometry. Flow Turbul. Combust. 104, 1029-1046 (2019)

Lazik, W., Doerr, T., Bake, S., v. d. Bank, R., Rackwitz, L.: Development of Lean-Burn Low-NOx Combustion Technology at Rolls-Royce Deutschland. Volume 3: Combustion, Fuels and Emissions, Parts A and B, pp. 797-807 (2008)

Maas, U., Bykov, V.: The extension of the reaction/diffusion manifold concept to systems with detailed transport models. Proc. Combust. Inst. 33(1), 1253-1259 (2011)

Maas, U., Pope, S.B.: Simplifying chemical kinetics: intrinsic low-dimensional manifolds in composition space. Combust. Flame 88, 239-264 (1992)

Matzen, M., Demirel, Y.: Methanol and dimethyl ether from renewable hydrogen and carbon dioxide: alternative fuels production and life-cycle assessment. J. Clean. Prod. 139, 1068-1077 (2016)

Neagos, A., Bykov, V., Maas, U.: Adaptive hierarchical construction of reaction-diffusion manifolds for simplified chemical kinetics. Proc. Combust. Inst. 36(1), 663-672 (2017)

Poinsot, T., Veynante, D.: Theoretical and Numerical Combustion, 3 edn. RT Edwards, Inc., (2011)

Popp, S., Hunger, F., Hartl, S., Messig, D., Coriton, B., Frank, J.H., Fuest, F., Hasse, C.: LES flamelet-progress variable modeling and measurements of a turbulent partially-premixed dimethyl ether jet flame. Combust. Flame 162, 3016-3029 (2015)

Saksena, M.P., Saxena, S.: Viscosity of multicomponent gas mixtures. In: Proceedings of the 6th Symposium on Thermophysical Properties, vol. 31, pp. 100-110 (1963)

Semelsberger, T.A., Borup, R.L., Greene, H.L.: Dimethyl ether (DME) as an alternative fuel. J. Power Sources 156, 497-511 (2006)

Smith, G.P., Golden, D.M., Frenklach, M., Moriarty, N.W., Eiteneer, B., Goldenberg, M., Bowman, C.T., Hanson, R.K., Song, S., Gardiner, W.C.J., Lissianski, V.V., Qin, Z.: GRI 3.0 reaction mechanism. http://www.me.berkeley.edu/grimech (1999). Accessed Oct 2019

Steinhilber, G., Bykov, V., Maas, U.: REDIM reduced modeling of flame-wall-interactions: quenching of a premixed methane/air flame at a cold inert wall. Proc. Combust. Inst. 36(1), 655-661 (2017)

Strassacker, C., Bykov, V., Maas, U.: REDIM reduced modeling of flame quenching at a cold wall-the influence of detailed transport models and detailed mechanisms. Combust. Sci. Technol. 191(2), 208222 (2018)

Strassacker, C., Bykov, V., Maas, U.: REDIM reduced modeling of quenching at a cold wall including heterogeneous wall reactions. Int. J. Heat Fluid Flow 69(January), 185-193 (2018)

Strassacker, C., Bykov, V., Maas, U.: Comparitive analysis of reaction-diffusion manifold based reduced models for head-on- and side-wall-quenching flames. In: Combustion Symposium (2019). Publication submitted

Tamura, M., Berg, P.A., Harrington, J.E., Luque, J., Jeffries, J.B., Smith, G.P., Crosley, D.R.: Collisional quenching of $\mathrm{CH}(\mathrm{A}), \mathrm{OH}(\mathrm{A})$, and $\mathrm{NO}(\mathrm{A})$ in low pressure hydrocarbon flames. Combust. Flame 114, 502-514 (1998)

van Oijen, J.A., Donini, A., Bastiaans, R.J., ten Thije Boonkkamp, J.H., de Goey, L.P.: State-of-the-art in premixed combustion modeling using flamelet generated manifolds. Prog. Energy Combust. Sci. 57, 30-74 (2016)

van Oijen, J.A., de Goey, L.P.: Modelling of premixed laminar flames using flamelet-generated manifolds. Combust. Sci. Technol. 161(1), 113-137 (2000)

Zhao, Z., Chaos, M., Kazakov, A., Dryer, F.L.: Thermal decomposition reaction and a comprehensive kinetic model of dimethyl ether. Int. J. Chem. Kinet. 40, 1-18 (2008)

Zirwes, T., Häber, T., Zhang, F., Kosaka, H., Dreizler, A., Steinhausen, M., Hasse, C., Suntz, R., Bockhorn, H., Trimis, D.: Numerical and Experimental Investigation of Chemiluminescent Radical Concentrations During Side-Wall Quenching. Flow, Turbulence and Combustion (2019). Publication submitted

Zschutschke, A., Messig, D., Scholtissek, A., Hasse, C.: Universal Laminar Flame Solver (ULF). figshare (2017). https://figshare.com/articles/ULF_code_pdf/5119855/2 